ISSN 0103-9954

\title{
AVALIAÇÃO DA PRODUÇÃO DE LÁTEX EM MANGABEIRAS DO CERRADO GOIANO
}

\author{
MAGABEIRA LATEX PRODUCTION EVALUATION IN CERRADO REGION OF GOIÁS
}

\author{
Alcione da Silva Arruda ${ }^{1}$ Rute Quelvia de Faria ${ }^{2}$ Nei Peixoto ${ }^{3}$ Ana Silvia Franco Pinheiro Moreira ${ }^{4}$ \\ Juliana Ferreira Floriano ${ }^{5}$ Carlos Frederico Oliveira Graeff ${ }^{6}$ Pablo José Gonçalves ${ }^{7}$ \\ Luciane Madureira de Almeida ${ }^{8}$
}

\begin{abstract}
RESUMO
O látex de mangabeira, planta nativa do cerrado, apresenta potencial biotecnológico para desenvolvimento de novos fármacos. Pouco se sabe sobre os métodos de coleta e produtividade de látex em mangabeira, a qual vem sendo explorada comercialmente para produção de frutos de modo principalmente extrativista. Buscando informações sobre a utilização do látex de mangabeira foi avaliada, nesse trabalho, a produtividade do látex associada aos métodos de sangria; às condições ambientais; às características sazonais e anatômicas dessa espécie. Como resultado observou-se que o método de coleta utilizado comumente em seringueira não é apropriado para mangabeira, sendo a sangria feita com facão na região do caule o método mais produtivo. Em relação à anatomia da casca, a espessura, a posição e o número de vasos lactíferos influenciam na produção. Resultados também mostraram que a casca de mangabeira é espessa e que o número de vasos lactíferos é superior na região interna, evidenciando que sangrias utilizando cortes superficiais não são eficientes, pois não atingem os vasos lactíferos. Também, foi observado que o aumento da temperatura ambiental tem influência positiva na produtividade do látex e que na época da frutificação a produção de látex é significativamente inferior. Isso provavelmente ocorre porque a planta utiliza uma parcela dos seus metabólitos secundários para formação do fruto, gerando uma menor disponibilidade desses metabólitos para a produção de látex.
\end{abstract}

Palavras-chave: Hancornia speciosa; extração de látex; características sazonais e anatômicas.

\section{ABSTRACT}

The mangabeira latex, plant native from Cerrado, shows biotechnological potential for the development of new drugs. Little is known about the latex productivity and bleeding methods in mangabeira, which is

1 Engenheira agrônoma, $\operatorname{Dr}^{\mathrm{a}}$., Professora da Unidade Universitária de Ipameri, Universidade Estadual de Goiás, Rodovia GO330, Km 241, Setor Universitário, CEP 75780-000, Ipameri (GO), Brasil. alcione.sarruda@gmail.com

2 Engenheira agrônoma, MSc., Professora do Instituto Federal Goiano, Campus Urutaí, Rodovia Geraldo Silva Nascimento Km 2,5, CEP 75790-000, Urutaí (GO), Brasil. rute_abu@yahoo.com.br

3 Engenheiro agrônomo, Dr., Professor da Unidade Universitária de Ipameri, Universidade Estadual de Goiás, Rodovia GO330, Km 241, Setor Universitário, CEP 75780-000, Ipameri (GO), Brasil. nei.peixoto48@gmail.com

4 Bióloga, Dra ${ }^{\text {. }}$, Professora do Departamento de Biologia Geral, Universidade Federal de Uberlândia, Av. Pará, 1720, Campus Umuarama, Caixa Postal 593, Uberlândia (MG), Brasil. anasilvia@inbio.ufu.br

5 Bióloga, Dra ., Pós-doutoranda do Departamento de Física da Faculdade de Ciências, Universidade Estadual Paulista Júlio Mesquita Filho, Campus Bauru, Avenida Luiz Edmundo Carrijo Coube, 14-01, Vargem Limpa, CEP 17033-360, Bauru (SP), Brasil. juli@ibb.unesp.br

6 Físico, Dr., Professor Titular do Departamento de Física da Faculdade de Ciências, Universidade Estadual Paulista Júlio Mesquita Filho, Campus Bauru, Av. Luiz Edmundo Carrijo Coube, 14-01, Vargem Limpa, CEP 17033-360, Bauru (SP), Brasil. graeff@fc.unesp.br

7 Físico, Dr., Professor Adjunto do Instituto de Física, Universidade Federal de Goiás, Campus Samambaia, Itatiaia, Caixa Postal 131, Goiânia (GO), Brasil. pablo@ufg.br

8 Bióloga, Drª., Professora da Unidade Universitária de Ipameri, Universidade Estadual de Goiás, Rodovia GO330, Km 241, Setor Universitário, CEP 75780-000, Ipameri (GO), Brasil. luciane.almeida@ueg.br

Recebido para publicação em 29/04/2013 e aceito em 13/06/2014

Ci. Fl., v. 26, n. 3, jul.-set., 2016 
usually utilized in extractive way for fruit production. Aiming to broaden the knowledge about mangabeira latex production it was evaluated the productivity associated with bleeding methods; environmental conditions; anatomical aspects from the bark and seasonal properties. As results it was observed that the bleeding method commonly used in rubber tree is not appropriated to mangabeira; the most productive bleeding method was using a knife in stalk region. The bark thickness and lactiferous vessels number are associated with latex production. Anatomical analysis showed that the bark in mangabeira is thick and the lactiferous vessels are deeply localized, in this way some superficial bleeding methods are not able to reach the vessels. It was also observed that the increase in environmental temperature has a positive influence on the productivity of latex and that during the fruiting time the latex production is significantly lower. This probably occurs because the plant uses part of the secondary metabolites for fruit formation, which reduced the availability of these metabolites to latex production.

Keywords: Hancornia speciosa; latex extraction; anatomical and seasonal characteristics.

\section{INTRODUÇÃO}

Embora milhares de espécies vegetais possuam valor terapêtico comprovado e sejam frequentemente empregadas na medicina tradicional, estima-se que apenas $1 \%$ delas seja cientificamente conhecido (PRIMACK, 1993). Nesse sentido, o uso de certas plantas na medicina popular pode ser um bom indicativo da potencialidade de novos medicamentos. Um produto vegetal que tem despertado interesse e já é comumente empregado para o tratamento de inúmeras enfermidades é o látex de algumas plantas. $\mathrm{O}$ látex representa $\mathrm{o}$ conteúdo citoplasmático de um sistema de células especializadas, conhecidas como laticíferos (AZZINI et al., 1998). As funções atribuídas ao látex nas plantas são as de proteção contra herbívoros e micro-organismos e de selar ferimentos (FARREL et al., 1991).

Estimativas indicam que existem de 12 a 35 mil espécies lactíferas com seu potencial farmacológico praticamente desconhecido (KONNO et al., 2004). Dentre elas, o mais conhecido e explorado comercialmente é o látex proveniente da seringueira, o qual vem sendo empregado para fabricação de borracha e diversos outros produtos comerciais. Recentemente foi descoberto que o látex de seringueira também apresenta uma surpreendente ação cicatrizante, a qual está associada ao seu potencial angiogênico (MRUÉ, 2000). Essas propriedades indutoras de regeneração tecidual do látex foram constatadas em diferentes estudos, tais como regeneração de ossos (ERENO et al, 2006, MARTINS et al, 2010); restituição de membrana timpânica (ARAUJO et al., 2012); neovaginoplastia (CARVALHO et al., 2008); restituição alvéolo dental de ratos (BALABANIAN et al., 2006); restituição da retina de coelho (SAMPAIO et al., 2010) entre outros. Apesar dos expressivos resultados obtidos, existem alguns relatos sobre alergias a produtos à base de látex de seringueira (EBO e STEVENS, 2002; YAGAMI et al., 2004). A alergia ocorre quando há uma sensibilização, pelo contato inicial com as proteínas do látex (BEEZHOLD et al., 1994). Esse é um dos motivos da busca por outras espécies lactíferas, as quais possam produzir látex com menor potencial alergênico. A mangabeira (Hancornia speciosa), planta nativa do cerrado e pouco explorada comercialmente, pode ser uma alternativa para extração e exploração de látex para utilização biomédica (ALMEIDA et al., 2014). Uma das vantagens do uso da mangabeira seria a possibilidade desta planta produzir um látex com propriedade angiogênica e com menor potencial alergênico, uma vez que a carga de proteínas do látex de mangabeira é inferior ao látex de seringueira (MALMONGE et al., 2009).

$O$ interesse na cultura da mangabeira vem crescendo nos últimos anos, sendo o principal interesse comercial o fruto, o qual é denominado mangaba. O mesmo pode ser consumido in natura ou processado como geleias, doces, sorvetes, sucos, refrescos, picolés, licores, vinho e xarope (SOUZA, 2001). Além dos frutos, outros importantes subprodutos com potencial farmacológico podem ser extraídos dessa planta. Por exemplo, as folhas são utilizadas para extração de substâncias ativas (esteroides, triterpenos e taninos) usadas para controle da pressão arterial, sendo esses constituintes 10 vezes mais eficientes que o captopril, remédio comumente usado para controle da pressão (FERREIRA et al., 2007 a,b; SILVA et al., 2011). A casca produz diferentes tipos de flavonoides, catequinas, antocianinas e taninos 
usados no tratamento de gastrite causada por Heliobacter pylori (MORAES et al., 2008). Ainda, na medicina popular o látex de mangabeira é usado para estimular funções hepáticas e tratar doenças dermatológicas, hipertensão e diabetes (MACEDO e FERREIRA, 2004; RITTER et al., 2002). Recentemente, Marinho et al. (2011) mostraram que o látex de mangabeira apresenta atividade antiinflamátoria a qual foi constatada através da inibição da formação de ácido nítrico, PGE2 e produção de citocinina.

A mangabeira é uma fruteira perenifólia de clima tropical (SOUZA, 2001) a qual vegeta espontaneamente em solos secos e arenosos adaptando-se a diferentes climas (MOURA et al., 2002). No Cerrado, a mangabeira ocorre principalmente em encostas, as quais geralmente são inapropriadas para agricultura (REZENDE et al., 2003). Sendo assim, a conservação e enriquecimento dessas áreas com mangabeiras poderiam representar uma boa alternativa para valorização desses ambientes e sua exploração racional e sustentada pelas populações locais (AGUIAR FILHO et al., 1998). Sua floração, na região dos cerrados, ocorre no período de agosto a novembro e sua frutificação pode ocorrer em qualquer época do ano, mas se concentra principalmente de julho a outubro ou de janeiro a abril (SOARES et al., 2006). De acordo com Lima e Scariot (2010), existe uma variação na produção de frutos por planta, sendo que há mangabeiras que podem produzir mais de 800 frutos em um ano.

Apesar do potencial econômico, existem poucos plantios comerciais de mangabeira no Brasil (LEDERMAN e BEZERRA, 2003) sendo que na maioria dos estados produtores, a produção de frutos que chega ao mercado e às indústrias de processamento é extrativista. Segundo dados do Instituto Brasileiro de Geografia e Estatística (2010), a produção de mangaba no Brasil decaiu entre os anos de 2004 (999 toneladas) a 2010 (722 toneladas).

Até o momento pouco é conhecido sobre a biologia dessa espécie, principalmente no que se diz respeito à produção de látex. Poucos trabalhos são encontrados na literatura sobre métodos de coleta e ferramentas adequadas para sangria do látex em mangabeira (LIMA e SCARIOT, 2010; BEKKEDAL e SAFFIOTI, 1947). Em relação à produtividade, não foram ainda realizados estudos sobre a influência da temperatura, umidade e precipitação pluviométrica na produção do látex; tampouco foi avaliada a influência das características anatômicas e sazonais das plantas na produção de látex. Por outro lado, todos esses estudos já foram realizados em seringueiras e se mostraram de grande importância na exploração comercial e melhoramento genético da espécie (GONÇALVES et al., 2000; MORENO et al., 2005; MESQUITA et al., 2006; COSTA et al., 2008, 2010; ZHENGQUAN et al., 2008; MESQUITA e OLIVEIRA 2010; SILVA et al., 2013). Procurando buscar informações sobre a exploração do látex de mangabeira, os seguintes aspectos foram avaliados nesse trabalho: a produtividade associada a três diferentes métodos de sangria; a produtividade associada às condições ambientais (temperatura, umidade e precipitação pluviométrica); e a produtividade associada às características sazonais e anatômicas.

\section{MATERIAL E MÉTODOS}

\section{Material biológico}

As amostras de látex foram coletadas em plantas da coleção de mangabeiras da Universidade Estadual de Goiás - UEG, no município de Ipameri, plantadas em 2003. O manejo da área foi realizado apenas com capina manual para controle de ervas daninhas e iscas atrativas para reduzir a incidência de formigas cortadeiras. Nenhum tipo de fertilizante ou estimulante foi empregado para aumento da produção de látex. As análises foram realizadas em Delineamento Inteiramente Casualizado, com 20 repetições representadas por cada árvore de mangabeira tendo cada uma em torno de 9 anos de idade.

\section{Seleção das mangabeiras utilizadas no estudo}

Foram realizadas medidas da altura e diâmetros do caule e ramos em 300 mangabeiras disponíveis na estação experimental da UEGIpameri. Dessas, foram selecionadas 20 árvores, com medidas similares, para os experimentos de produtividade. As árvores possuíam altura média da bifurcação do caule (PIN - ponto de bifurcação do caule) de $67,5 \pm 25,3 \mathrm{~cm}$, diâmetro médio do caule de $36 \pm 3,5 \mathrm{~cm}$ e diâmetro médio dos ramos de 16,4 $\pm 1,2 \mathrm{~cm}$.

\section{Método de sangria}

Foram usados três sistemas de sangrias, 
chamados de métodos $\mathrm{S}, \mathrm{F}$ e R. O método $\mathrm{S}$ foi similar ao realizado em seringueira com corte em meia espiral na região do caule na altura aproximada de $30 \mathrm{~cm}$ do solo, utilizando faca específica de sangria em seringueira. No método F o corte vertical (10 $\mathrm{cm}$ de comprimento e profundidade de $0,5 \mathrm{~cm}$ ) foi realizado com o uso de um facão de acordo com as recomendações de moradores da região que extraem látex para uso na medicina popular. Para o método $\mathrm{R}$ foi realizado o corte vertical com faca na região dos ramos, cerca de 3 a $5 \mathrm{~cm}$, na profundidade de $0,5 \mathrm{~cm}$.

\section{Avaliação da produção de látex}

A produtividade de cada planta foi aferida de acordo com o volume de látex obtido com as sangrias nos diferentes experimentos. As sangrias foram realizadas uma vez por mês ao longo de um ano, as mesmas foram realizadas em dia sem chuva, iniciada às seis horas da manhã e finalizada em torno das oito horas da manhã. A temperatura, umidade do ar e precipitação pluviométrica foram avaliadas mensalmente. Também foram avaliadas as características fenológicas (presença e ausência de frutos) das plantas durante as coletas. O armazenamento do látex foi feito em tubos falcon de $50 \mathrm{ml}$. Não foram adicionados anticoagulantes nos recipientes de coleta do látex até que o mesmo fosse processado no laboratório.

\section{Anatomia da casca de mangabeira}

O estudo anatômico da casca da mangabeira foi realizado ao final de um ano de coleta. O material foi obtido através do corte feito na casca a uma altura de $60 \mathrm{~cm}$ do solo, chegando até o xilema da planta. Foram feitos cortes transversais para medição da espessura da casca, a qual foi realizada com auxílio de um paquímetro.

Os cortes longitudinais tangenciais foram feitos para avaliar número de vasos laticíferos e diâmetro dos mesmos. Todos os cortes foram realizados à mão livre, com o auxílio de uma lâmina de barbear e as seções foram coradas com Safrablau (solução de azul de astra e safranina na proporção 9:1).

Para análise dos vasos laticíferos foram determinadas duas regiões para avaliações, uma é referente à região próxima ao xilema - região interna, e a outra na região próxima à casca - região externa. Foram analisados número de vasos por planta, número médio de vasos por $\mathrm{mm}^{2}$ e diâmetro dos mesmos nas diferentes regiões, segundo a metodologia descrita por Laboriau et al., (1961). Para obtenção dos resultados de número de vasos laticíferos, calculou-se a média aritmética de cinco repetições para cada planta, analisando com objetiva no aumento de 10X. O diâmetro dos vasos laticíferos foi avaliado no aumento de 40X, sendo medidos em três pontos distintos para obtenção da média. As observações e fotos foram tiradas em microscópio Modelo Zeiss Germany Axyoscope.

\section{Análise estatística}

Para todos os experimentos foram realizadas a estatística descritiva básica com a obtenção dos valores médios de produção de látex e seus respectivos desvios padrões. Para a avaliação do método de sangria mais adequado para a mangabeira foi adotada estatística descritiva para dados não paramétricos, utilizando o Teste de Kruskal-Wallis em nível de significância de 5\% pelo Teste t (SPRENT e SMEETON, 2007). As variáveis estudadas foram os três métodos de sangria $\mathrm{F}, \mathrm{S}$ e $\mathrm{R}$ versus a produção anual de látex de cada árvore. $\mathrm{Na}$ avaliação de possíveis diferenças na quantidade e diâmetro dos vasos lactíferos das regiões internas e externas da casca de mangabeira foi aplicado o Teste de Wilcoxon em nível de significância de 5\% (SPRENT e SMEETON, 2007). As variáveis comparadas foram o número de vasos da região interna versus número de vasos da região externa; e diâmetro dos vasos internos versus diâmetro dos vasos externos. A relação entre produção de látex e fatores climáticos e fenológicos foi avaliada pelo Teste de Correlação de Pearson. As variáveis analisadas foram temperatura, umidade relativa do ar, precipitação e frutificação. Estas variáveis foram correlacionadas à produção anual de látex de cada árvore estudada pelo método de coleta $\mathrm{F}$. $\mathrm{O}$ índice de correlação foi testado ao nível de significância de $5 \%$ pelo Teste $t$.

\section{RESULTADOS E DISCUSSÃO}

Existem poucas informações sobre a produtividade de látex na espécie Hancornia speciosa, por isso avaliou-se nesse trabalho a produtividade de látex em relação a: 1) métodos de coleta, 2) características anatômicas, 3) características ambientais (temperatura, umidade e precipitação pluviométrica); e. 4) características 
sazonais da planta.

Avaliação de métodos de sangria para coleta do látex

Os métodos de sangria $\mathrm{S}, \mathrm{F}$ e $\mathrm{R}$ foram comparados em relação à produtividade média em mililitros ao longo de 12 coletas (Figura 1, Tabela 1), sendo realizada uma coleta por mês, iniciada em agosto de 2011 e finalizada em julho de 2012.

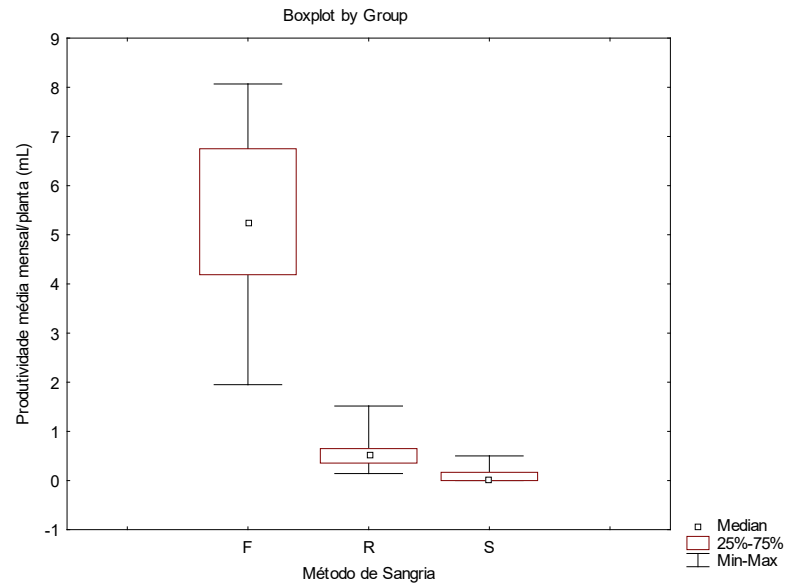

Em que: $\mathrm{S}=$ corte semelhante ao de seringueira em meia espiral; $\mathrm{F}=$ corte vertical com facão na região do caule; $\mathrm{R}=$ corte vertical com faca na região dos ramos.

FIGURA 1: Produtividade de látex de Hancornia speciosa nos diferentes métodos de sangria.

FIGURE 1: Latex production of Hancornia speciosa using different extraction methods.

TABELA 1: Comparação da produção média de látex obtida pelos diferentes métodos de coleta usando o teste de KruskalWallis $(\mathrm{p}<0,05 \%)$.

TABLE 1: Mean latex production comparison obtained by different collection methods using the Kruskal-Wallis test $(\mathrm{p}<0,05 \%)$.

\begin{tabular}{ccc}
\hline Método de sangria & Média $^{* *}$ & Classific. \\
\hline $\mathrm{F}$ & 5,39 & $\mathrm{a}$ \\
$\mathrm{R}$ & 0,58 & $\mathrm{~b}$ \\
$\mathrm{~S}$ & 0,09 & $\mathrm{c}$ \\
\hline
\end{tabular}

Em que: $\mathrm{S}=$ corte semelhante ao de seringueira em meia espiral; $\mathrm{F}$ = corte vertical com facão na região do caule; $\mathrm{R}$ - corte vertical com faca na região dos ramos.
O teste estatístico de Kruskal-Wallis foi aplicado para avaliar se existem diferenças de produtividade entre as metodologias de sangria avaliadas. O método com facão na altura do caule (F) foi o mais eficiente na coleta do látex, com a produção quase 10 vezes superior à extração na região dos ramos $(\mathrm{R})$, sendo, portanto, o método $\mathrm{F}$ o mais indicado para mangabeira (Figura $1 \mathrm{e}$ Tabela 1). Lima e SCARIOT (2010) relatam que o corte horizontal com facão na região do caule é comumente usado por extrativistas para coleta do látex, porém, acredita-se que se esse corte for extenso e atingir o floema pode prejudicar a planta, por isso nesse trabalho optou-se pelo corte vertical na altura do tronco. Outro motivo para mudar a direção do corte é a maior facilidade para coleta do látex. Estudos mais detalhados sobre métodos de coletas em mangabeira devem ser realizados com o objetivo de diminuir a área de ferimento da planta, aumentar a taxa de regeneração da casca e desenvolver ferramenta específica para extração de látex nessa espécie. Esse tipo de trabalho foi realizado em seringueira, a qual era inicialmente sangrada com cortes descontínuos feitos em forma de risco a uma distância de $2 \mathrm{~cm}$ e o qual atualmente evoluiu para cortes declivosos com a remoção de uma fina camada de casca usando ferramenta específica (DUARTE, 2007)

A produtividade utilizando o método "S" foi significativamente menor que nos outros tratamentos, indicando que o método de sangria comumente aplicado em seringueira é impróprio para mangabeira. $\mathrm{O}$ volume de látex obtido nas sangrias realizadas no presente estudo foi muito baixo em relação a alguns dados da literatura. Bekkedal e Saffioti (1947) afirmam que mangabeiras adultas, corretamente sangradas, em uma ou duas horas de escorrimento, produzem um litro de látex por sangria, produção equivalente à da seringueira. Contudo, Bekkedal e Saffioti (1947) avaliaram uma variedade de mangabeira (amazônica) diferente da utilizada nesse experimento.

\section{Avaliação da anatomia da casca de mangabeira}

A mangabeira, como a maioria das plantas do cerrado, apresenta caule aéreo muito espesso e com acúmulo de cortiça para proteção contra o fogo (SCARIOT et al., 2005). Na casca de mangabeira, como na maioria dos órgãos de representantes da família Apocynaceae existe a presença de vasos lactíferos (METCALFE, 1967). Contudo, esses 
vasos lactíferos são distinguíveis entre os diferentes órgãos da planta, devido ao seu diâmetro interno, conteúdo, espessura das paredes e ramificações do sistema (DEMARCO et al., 2006). Mesquita e Oliveira (2010) relatam que a organização estrutural da casca e a variabilidade das plantas em relação às características dos vasos laticíferos, podem ser fatores importantes na busca de uma maior compreensão dos mecanismos envolvidos na produção de látex. Assim, informações sobre a espessura da casca e localização dos vasos lactíferos são essenciais para melhorar os métodos de coleta e aumentar a produção de látex.

A espessura média da casca da mangabeira obtida nesse trabalho foi de $8 \mathrm{~mm}$, sendo de maior espessura que a casca de seringueira, $0,72 \mathrm{~mm}$ (MESQUITA e OLIVEIRA, 2010). Em estudo sobre espessura da casca, Gomez (1982) verificou que a profundidade da incisão tem estreita relação com a produção de látex em seringueira, pois o maior escoamento de látex está relacionado com os cortes mais profundos até a distância de 1 a $1,5 \mathrm{~mm}$ do câmbio. Por outro lado, incisão superior a este limite resulta em lesões das camadas regenerativas do câmbio, provocando o surgimento de nodosidades que, dependendo da extensão e quantidade, tornam a casca irregular, dificultando ou impossibilitando a sangria no ciclo seguinte. Essas nodosidades foram detectadas no método de coleta utilizado nesse trabalho (método F) e dificultaram a extração de látex na região próxima ao corte (Figura 2).

Com relação aos vasos lactíferos, de acordo com Pinheiro (2003), a mangabeira possui o sistema lactífero de vasos inarticulados e cada incisão no tronco, em termos médios, é capaz de esgotar uma área bem limitada de apenas $13 \mathrm{~cm}$ acima e $5 \mathrm{~cm}$ abaixo da superfície do corte. Como o sistema de vasos é restrito, exige tempo para recompor o látex e permitir outra sangria (PINHEIRO, 2003).

As análises histológicas dos vasos mostraram que nas diferentes regiões em estudo, região interna e externa, o número médio de vasos/ $\mathrm{mm}^{2}$, apresentou diferença significativa (Teste de Wilcoxon $\mathrm{z}=3,69$ e $\mathrm{p}<0,01 \%$ ), ocorrendo em maior frequência na região interna (Tabela 2, Figura 2), porém, em relação ao diâmetro médio dos vasos, não houve diferença significativa. Assim, pode-se concluir que a profundidade do corte é de fundamental importância para coleta de látex em mangabeira, visto que cortes superficiais não atingem grande quantidade de vasos lactíferos.

\section{Avaliação da produtividade em relação a características ambientais (temperatura, umidade e precipitação pluviométrica)}

A produção de látex é dependente dentre outros fatores, da disponibilidade de carboidratos de reserva, do estado nutricional da planta, da estrutura anatômica de vasos lactíferos e da biossíntese de aminoácidos e proteínas (MELO et al., 2006). Além desses fatores, as mudanças climáticas sazonais que envolvem flutuações na disponibilidade de luz, temperatura e umidade do ar afetam a atividade
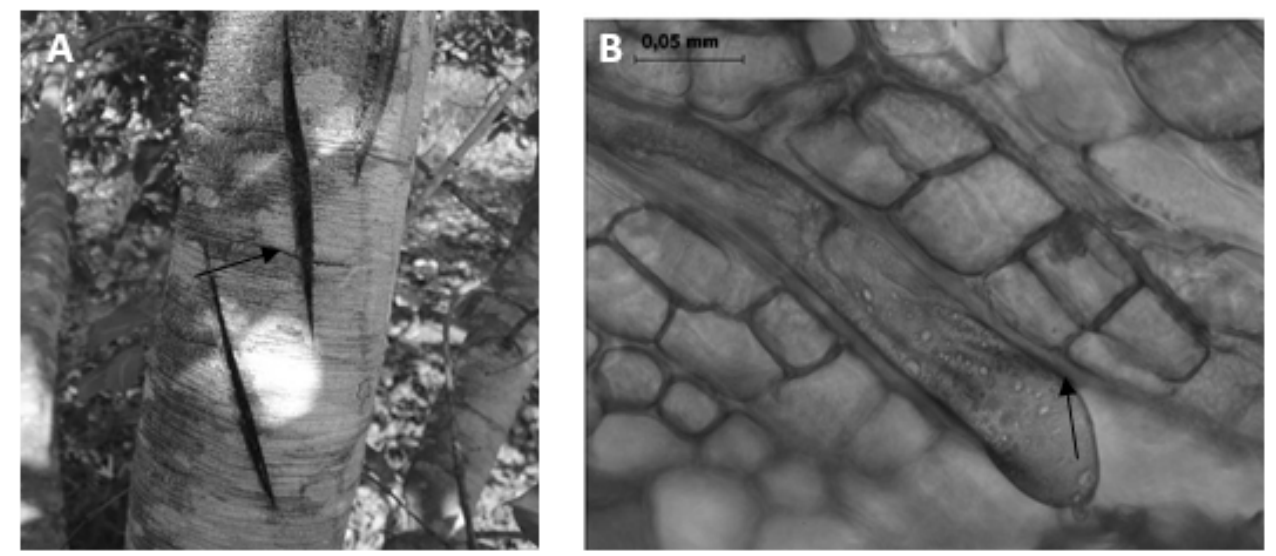

FIGURA 2: A) Nodosidades geradas na casca de mangabeira devido à extração de látex. B) Corte longitudinal tangencial da casca de mangabeira. Seta indica vaso laticífero com presença de látex (aumento de 40X).

FIGURE 2: A) Bark nodule originated by latex extraction. B) Tangential longitudinal section from mangabeira bark. A lactiferous vessel with latex content is indicated by arrow, magnification: $40 \mathrm{X}$. 
TABELA 2: Resultados do número médio de vasos e diâmetros, em $\mathrm{mm}^{2}$, e parâmetros estatísticos obtidos pelo teste não paramétrico de Wilcoxon.

TABLE 2: Mean number of vessels and their average diameters, in $\mathrm{mm}^{2}$, and statistical non parameters test (Wilcoxon).

\begin{tabular}{ccccc}
\hline Variável analisada & Interno $( \pm \mathrm{SD})$ & Externo $( \pm \mathrm{SD})$ & $\mathrm{Z}$ & $\mathrm{P}$ \\
\hline Número médio de vasos & $2,2( \pm 1,20)^{* *}$ & $1,68( \pm 0,89)$ & 3,69 & 0,0001 \\
Diâmetro médio dos vasos & $47,45( \pm 19,25)$ & $50,72( \pm 20,94)$ & 0,70 & 0,48 \\
\hline
\end{tabular}

Em que: SD: desvio padrão; Z: aproximação da distribuição binomial pela normal; P: probabilidade associada de ocorrência; $* *$ significativo ao nível de $1 \%$.

fotossintética e, consequentemente, o metabolismo da planta, alterando a produção de látex (PUSHPARAJAH, 1980; ORTOLANI et al., 1996; MELO et al., 2004). Por essa razão, avaliaram-se as condições climáticas nos meses de coletas, que foram correlacionadas com a produtividade de látex, obtida pelo método (F). De acordo com a Tabela 4, os resultados mostram que a temperatura média mensal apresentou uma correlação significativamente positiva com a produção de látex. As maiores produções ocorreram nos meses com as temperaturas mais elevadas (janeiro a abril), nos quais a média mensal de produção chegou a $166,4 \mathrm{~mL}$ de látex coletado. Já os menores índices de produção, ocorreram nos meses mais frios (maio a julho), com média de produção mensal de $50,9 \mathrm{~mL}$ de látex, por coincidir com o período de frutificação da espécie, que apresentou uma correlação negativa com a produção.

Os resultados são similares aos obtidos por Souza (2001), que observou que a mangabeira apresenta maior desenvolvimento vegetativo nas épocas de temperatura mais elevada e que a pluviosidade ideal está entre $750 \mathrm{~mm}$ e $1.600 \mathrm{~mm}$ anuais. $\mathrm{O}$ mesmo foi obtido para seringueira por Moreno et al. (2005), os quais mostraram que o pico máximo de produção de látex ocorre em fevereiro e o pico mínimo em junho.

Estudos realizados para verificar a produção de látex em seringueira mostram que a umidade e pluviosidade são fatores que têm correlação positiva com a produção de látex de alguns clones, mas existem exceções (MESQUITA et al., 2006; MELO et al., 2004; GONÇALVES et al., 1991). No presente estudo, tanto a umidade quanto a pluviosidade, apresentaram baixa correlação com a produção de látex em mangabeira.

\section{Avaliação da produtividade em relação a características sazonais}

Durante o processo de frutificação a planta utiliza uma parcela dos seus metabólitos secundários para formação do fruto, gerando uma menor disponibilidade desses metabólitos para a produção de látex. Uma classe de metabólitos secundários presente em grande quantidade no látex são os terpenos, os quais compõem os poli-isoprenos, principais compostos da borracha (PERES, 2004). Muitos compostos vegetais são terpenos ou possuem derivados de terpenos em sua molécula. Entre esses compostos encontram-se, inclusive, quatro das seis principais classes de hormônios vegetais (PERES, 2004). Para identificar se as características sazonais influenciavam na quantidade de látex produzido, os índices de produção foram correlacionados à presença e ausência de frutos (Tabela 4).

O teste de Correlação de Pearson indicou que existe uma forte correlação negativa entre a presença de frutos e a produção de látex $(-0,87)$, pois as menores produtividades ocorreram nos meses de maio a julho, época de frutificação no

TABELA 4: Coeficiente de correlação de Pearson, para as variáveis comparadas.

TABLE 4: Pearson correlation coefficient for the variables compared.

\begin{tabular}{lcccc}
\hline & PF & TMm & URm & Pm \\
\hline Produção de látex & $-0,87$ & 0,79 & 0,27 & 0,49 \\
\hline $\begin{array}{l}\text { Em que: } P F=\text { Presença de frutos, } \\
\text { Precipitação mensal. }\end{array}$
\end{tabular}


ano de 2011. Assim, a produtividade de látex é diminuída significativamente na presença de frutos, isso porque a disponibilidade de metabólitos secundários necessários para produção do látex é reduzida durante o período de frutificação.

\section{CONCLUSÕES}

Os resultados apresentados nesse trabalho mostraram que fatores morfológicos, ambientais e sazonais estão fortemente associados à produção do látex em mangabeira. Em relação às características sazonais, na época de frutificação, existe uma redução significativa da produção de látex. Fatores ambientais também influenciam na produção, sendo a temperatura média mensal o mais significativo. Devido à maior espessura da casca, o método tradicionalmente empregado para coleta de látex em seringueiras não se mostrou eficiente para mangabeiras e então outro método foi proposto. Entretanto, apesar de mais eficiente, este método provocou nodosidades na casca, o que pode comprometer futuras sangrias naquela região. Os resultados apresentados neste trabalho sugerem ainda que há muito a ser investigado e explorado para o completo entendimento da biologia e fisiologia de mangabeira, bem como para a melhoria da produção de látex visando à exploração comercial sustentável. Esse trabalho é um primeiro relato sobre os fatores que influenciam a produção de látex em mangabeira e é importante como base para futuras análises visando ao melhoramento genético e aumento da produção de látex da espécie.

\section{AGRADECIMENTOS}

Essa pesquisa foi financiada pelos seguintes órgãos de fomento a pesquisa MCT/CNPq/FNDCT/ FAPEG/MEC/CAPES, no edital Centro-Oeste.

\section{REFERÊNCIAS BIBLIOGRÁFICAS}

AGUIAR FILHO, S.P.; BOSCO, J.; ARAÚJO, I.A. A mangabeira (Hancornia speciosa) domesticação e técnica de cultivo, João Pessoa: EMEPA, PB, 1998, 26 p. (EMEPA-PB. Documento, 24).

ALMEIDA, L.M. et al. Hancornia speciosa látex for biomedical application: physical and chemical properties, biocompatibility assessment and angiogenicactivity. Jornal of Material Science: Materials in Medicine, DOI 10.1007/s10856-0145255-8, 2014.
ARAÚJO, M.M.; MASSUDA, E.T.; HYPPOLITO, M.A. Anatomical and functional evaluation of tympanoplasty using a transitory natural latex biomembrane implant from the rubber tree Hevea brasiliensis. Acta Cirúrgica Brasileira, São Paulo, v. 27 (8), p 566-571, 2012.

AZZINI, A.; GONÇALVES, P.S.; TOMAZ, R.M.A.G. Sieve tubes diameter and the rubber production in rubber tree clones. Bragantia, v.57(1), p. 57-60, 1998.

BALABANIAN, C.A. et al. Biocompatibility of natural latex implanted into dental alveolus of rats. Journal of Oral Science, Matsuda, v. 48, p. 201-205, 2006.

BEEZHOLD, D.H. et al. A identificação de uma proteína alergênica $46-\mathrm{kD}$ látex em profissionais da saúde. Clinical Experimental Immunol., United Kingdom, v. 98 (3), p. 408-413, 1994.

BEKKEDAHAL, N.; SAFFIOTI, W. Mangabeira látex and rubber. The Rubber Age. Vol. 60, n. 5, p. 553-561, 1947.

CARVALHO, B.R. et al. Natural latex (Hevea brasiliensis) mold for neovaginoplasty. Rev Bras. Ginecol. Obstet., Rio de Janeiro, v.30 (1), p. 31-35, 2008.

COSTA, R.B. et al. Seleção simultânea para porte reduzido e alta produção de látex em seringueira. Bragantia, Campinas, v.67, n.3, p. 649-654, 2008. COSTA, R.B. et al. Predição de parâmetros e valores genéticos para caracteres de crescimento e produção de látex em progênies de seringueira. Bragantia, Campinas, v. 69, p. 49-56, 2010.

DEMARCO, D.; KINOSHITA, L.S.; CASTRO, M.M. Laticíferos articulados anastomosados novos registros para Apocynaceae. Revista Brasil. Bot., v. 29, p. 133-144, 2006.

DUARTE, J.D. Seringueira: da implantação ao beneficiamento primário. Valença. $1^{\circ}$ edição, 2007, $62 \mathrm{p}$.

EBO, D.G., STEVENS, W.J. IgE-mediated natural rubber látex allergy: an update. Acta Clin. Belg., Belgica, v. 57, p. 58-70, 2002.

ERENO, C. et al. Latex use as an occlusive membrane for guided bone regeneration. J. Oral Sci., Matsuda, v.48(4), p. 201-205, 2006.

FARREL, B.D.; DUSSOURD, D.E.; MITTER, C. Escalation of plant defense: do latex/resin canals spur plant diversification? American Naturalist, v. 138, p. 881-900, 1991.

FERREIRA, H.C. et al. Endothelium-dependent vasodilatation induced by Hancornia speciosa in rat superior mesenteric artery. Phytomedicine. 
Germany, v. 14, p. 473-478, 2007b.

FERREIRA, H.C. et al. Nitric oxide-dependent vasodilatation by ethanolic extract of Hancornia speciosa via phosphatidyl-inositol 3-kinase. Journal of Ethnopharmacology, Copenhagen, v. 109, p. 161-164, 2007a.

GONÇALVES, P.S. et al. Clones de Hevea: Influência dos fatores ambientais na produção e recomendação para plantio. Campinas: Instituto Agronômico de Campinas, 1991. 32p. (Boletim Técnico, 138).

GONÇALVES, P.S. et al. Efeito da frequência de sangria e estimulação no desenvolvimento produtivo e econômico de clones de seringueira. Pesquisa Agropecuária. Brasileira, Brasília, v. 35, n.6, p. 1081-1091, 2000.

GOMEZ, J. Anatomy of Hevea and it's influence on latex production. Kuala Lumpur: Malaysia Rubber Research and Development Board, 1982, $76 \mathrm{p}$.

INSTITUTO BRASILEIRO DE ESTATÍSTICA (IBGE) Disponível em: http:||www.ibge.gov.br. Acesso em: 10 de outubro de 2013.

KONNO, K. et al. Papain protects papaya trees from herbivorous insects: role of cysteine proteases in latex. Plant J., v. 37, p. 370-378, 2004.

LABOURIAU, L.G.; OLIVEIRA, J.G. SALGADOLABOURIAU, M.L. Transpiration of Schizolobium parahyba (Vell) Toledo I. Behavior in the rainy season, conditions Caeté, Minas Gerais. Anais da Academia Brasileira de Ciências, Manaus, v. 33(2), p. 237-257, 1961.

LEDERMAN, I.E.; BEZERRA, J.E.F. Situação atual e perspectivas da cultura de mangaba no Brasil. In: Simpósio Brasileiro sobre a cultura da Mangaba. Anais, Aracajú, 2003.

LIMA I.L.P.L., SCARIOT A. Boas práticas de manejo e extrativismo sustentável da mangabeira. EMBRAPA, Brasília, 64p, 2010.

MACEDO M, FERREIRA A.R. Plantas medicinais usadas no tratamento dermatológico da Bacia do alto Paraguai, Mato Grosso. Rev. Bras. Farmacongn., Curitiba, v. 14, p. 40-44, 2004.

MALMONGE, J.A. et al. Comparative study on technological properties of látex and natural rubber from Harconia speciosa gomes and Hevea brasiliensis. Journal of Applied Polymer Science, USA, v. 111, p. 2986-2991, 2009.

MARINHO D. $G$ et al. The latex obtained from Hancornia speciosa Gomes possesses anti-inflammatory activity. Journal of Ethnopharmacology. v. 135, p. 530-537, 2011.
MARTINS, R. et al. Comparative study of boné response guides tissue regeneration technique macroscopic evaluation, Part 1. FULL Densitry in Science, São José dos Pinhais, v. 1(3), p. 224-230, 2010.

MELO, R.F. et al. Variação sazonal de algumas características nutricionais e bioquímicas relacionadas com a produção de látex em clones de seringueira [Hevea brasiliensis (Wild.) Muell. Arg.] em Lavras -MG. Cienc. Agrotec., v. 28, p. 1326-1335, 2004.

MESQUITA, A.C. et al. Sazonalidade da produção e características do látex de clones de seringueira em Lavras, MG., Bragantia, Campinas, v.65, n4, p. 633-639, 2006.

MESQUITA, A.C.; OLIVEIRA, L.E.M. Características anatômicas da casca e produção em plantas de seringueira não enxertadas. Acta Amazonica, Manaus, vol. 40(2), p. 241-246, 2010. METCALFE, C.R. Distribution of latex in plant kingdom. Economic Botany, v.21, p. 115-127, 1967.

MORAES, T.M. et al. Hancornia speciosa: Indications of gastroprotective, healing and anti-Heliobacter pilori actions. Journal of Ethnopharmacology, Copenhagen, v. 120, p. 161-168, 2008.

MORENO, R.M.B et al. Technological properties of látex and natural rubber of Hevea brasiliensis clones. Sci. Agric., Piracicaba, v.62, n.2, p.122-126, 2005.

MOURA, CFH. Quality of fruits native to latin America for processing: mangaba (Hancornia speciosa Gomes). Acta Horticulture, Leuven, v. 2, n.575, p. 549-554, 2002.

MRUÉ, F. Neoformação tecidual induzida por biomembrana de látex natural com polilisina. Aplicabilidade na neoformação esofágica e da parede abdominal. Estudo experimental em cães. 2000. 112f. Tese (Doutorado em Cirurgia Veterinária), Faculdade de Medicina de Ribeirão Preto, Universidade de São Paulo, Ribeirão Preto, 2000.

ORTOLANI, A. A. et al. Agrometeorological models to estimate annual and seasonal production of látex in rubber. Rev. Bras. de Agrometeorologia, Sete Lagoas, v. 4(1), p. 147-150, 1996.

PERES, L.E.P. Metabolismo secundário. São Paulo: Escola Superior de Agricultura Luiz Queiroz, p. 26, 2004.

PINHEIRO, E. O latex e a borracha da mangabeira. In: Simposio Brasileiro sobre a cultura da mangaba, 
2003, Aracaju, SE. Embrapa Tabuleiros Costeiros, 2003.

PRIMACK, R. B. Essentials of conservation biology. Massachusetts: Sunderland, 1993. 546 p. PUSHPARAJAH, E. Problems and potentials for establishing Hevea under difficult environmental conditions. Planter, Kuala Lumpur, v. 50, p. 242-251, 1980.

REZENDE, C.F.A. et al. Caracterização de ambientes com alta densidade e ocorrência natural de mangabeira (Hancornia speciosa Gomes) no Cerrado. In: Simpósio Brasileiro sobre a cultura da mangaba, 2003, Aracaju, SE. Aracaju: Embrapa Tabuleiros Costeiros, 2003.

RITTER, M.R. et al. Plantas usadas como medicinais no município de Ipê, RS, Brasil. Rev. Bras. Framacogn., Curitiba, v12, p.51-62, 2002.

SAMPAIO, R.B. et al. Rabbit retinal neovascularization induced by latex angiogenicderived fraction: an experimental model. Curr. Eye Res., London, v.35(1), p.56-62, 2010.

SCARIOT, A. SOUZA-SILVA, J.C.; FELFILI, J.M. (ORG). Cerrado: ecologia, biodiversidade e conservação. Brasília, Ministério do Meio Ambiente, 2005.

SILVAC.G. etal. Hancornia speciosa Gomes induces hypotensive effect through inhibition of ACE and increase on NO. Journal of Ethnopharmacology. Copenhagen, v. 137, p. 709-711, 2011.

SILVA, G.A.P. et al. Genetic parameters and correlation in early measurement cycles in rubber trees. Euphytica, New York, v.189, p. 343-350, 2013.

SOARES, F. P. et al. Cultura da mangaba (Hancornia speciosa Gomes). Boletim Agropecuário, Lavras/ MG, n.67, p.1-12, 2006.

SOUZA, V.A.B. Perspectivas do melhoramento de espécies nativas do nordeste brasileiro. In: Congresso brasileiro de melhoramento genético de plantas, 1, 2001, Goiânia. Resumo 25, Embrapa Meio-Norte, Teresina - PI, 2001.

SPRENT, P.; SMEETON, N.C. Applied nonparametric statistical methods. Boca Raton: Chapman \& Hall. 2007, 530p.

YAGAMI, T. et al. Proteomic analysis of putative látex allergens. Int. Arch of Allergy and Immunology., Viena, v.135, p. 3-11, 2004.

ZHENG-QUAN, G.; XIAO, M.C.; e HAO, N. Y., Latex production with depolymerizing compoundas of actin cytoskeleton in rubber trees. Pesquisa Agropecuária Brasileira., Brasília, v. 43, n. 2, p. 275-279, 2008. 